www.ijpsonline.com

\title{
Development and Validation of a HPTLC Method to Determine Serum Zonisamide levels for Therapeutic Drug Monitoring in Clinical Settings
}

\author{
RENUKA P. MUNSHI* AND NAMRATA GAWDE \\ Department of Clinical Pharmacology, TNMC \& BYL Nair Hospital, Dr AL Nair Road, Mumbai-400 008, India
}

Munshi and Gawde: Development and Validation of a HPTLC method to Determine Serum Zonisamide

Levels

\begin{abstract}
This investigation is aimed to develop and validate a high-performance thin layer chromatography method for quantitative determination of serum zonisamide levels. Chromatographic separation was carried out on a silica gel 60F254 plate using a mixture of ethyl acetate:methanol:toluene $(4: 1: 5)$ as the mobile phase. Densitometric detection was carried out at $254 \mathrm{~nm}$. The method was validated for linearity, precision, selectivity, limit of detection, limit of quantification and accuracy. Linear calibration curves in the range of 5 to $80 \mu \mathrm{g} / \mathrm{band}$ gave a correlation coefficient of 0.991 . The intra-day $(n=6)$ and inter-day $(n=18)$ precision, expressed as the relative standard deviation were in the range of 2.83 to $3.27 \%$ and from 2.09 to $4.39 \%$. The limits of detection and quantification were found to be 1.07 and $3.15 \mu \mathrm{g} / \mathrm{band}$, respectively. Accuracy was calculated as percent recovery and was found to be 97.52 and $115.23 \%$. Theophylline was used as an internal standard, which gave a well separated peak at $R_{\mathrm{f}} \mathbf{0 . 3 6}$ without interfering with zonisamide. The method was found to be specific with no matrix interference. Thus, the method developed for the estimation of serum zonisamide level is simple, cost-effective and reliable for therapeutic drug monitoring.
\end{abstract}

Key words: Therapeutic drug monitoring, Antiepileptic, zonisamide, theophylline, HPTLC

Zonisamide is a 1,2-benzisoxazole derivative, a new generation antiepileptic drug, which suppresses the epileptogenic focus and blocks the spread or propagation of seizure discharges ${ }^{[1,2]}$. It is used in the treatment of simple and complex partial seizures, refractory partial seizures, generalized tonic-clonic, generalized clonic and infantile spasms ${ }^{[1-6]}$. Prevention and management of seizures requires continuous maintenance of effective levels of the antiepileptic drugs in the body. Due to pharmacokinetic variability and interaction with other antiepileptic drugs, there can be issues of either over-dosing resulting in toxicity, side effects like drowsiness, ataxia or under-dosing with zonisamide ${ }^{[1,2]}$. Hence it becomes imperative to perform regular monitoring of zonisamide level in patients.

Different approaches have been proposed for the determination of zonisamide such as high performance liquid chromatography, gas chromatography and micellar electrokinetic capillary chromatography ${ }^{[7-10]}$. However, all these techniques are not only expensive but also time-intensive. Hence, although therapeutic drug monitoring is important for this newer antiepileptic drug, not every patient can afford the costs required to carry out regular monitoring; therefore it was proposed to develop a method, which would be simple, reliable and cost-effective with a quicker turnaround time.

Zonisamide was kindly donated by Sun Pharma (India). Theophylline was purchased from Sigma Aldrich (India). Solvents and diluents used such as ethyl acetate, methanol, toluene and acetonitrile were of analytical grade, purchased from Merck (India). The chromatographic system used for the development and validation of the method was purchased from Camag (Switzerland) consisted of Linomat V applicator, TLC scanner IV, twin trough chambers, $100 \mu$ l Hamilton syringe and WinCats Software (version 1.4.9). TLC aluminum plates coated with silica gel 60 F254 were from Merck (Germany). Other equipment used were, nitrogen gas evaporator from Zymark Turbovap (USA) and a refrigerated centrifuge.

This is an open access article distributed under the terms of the Creative Commons Attribution-NonCommercial-ShareAlike 3.0 License, which allows others to remix, tweak, and build upon the work non-commercially, as long as the author is credited and the new creations are licensed under the identical terms

Accepted 05 February 2019

Revised 06 September 2018

Received 07 May 2018

*Address for correspondence

E-mail: renuka.munshi@gmail.com

March-April 2019

Indian Journal of Pharmaceutical Sciences 
High performance thin layer chromatographic (HPTLC) technique was carried out using HPTLC aluminum plates coated with silica gel. The plates were activated prior to application, by washing with methanol and further heating at $110^{\circ}$. The application of samples and standards on the activated HPTLC plate was carried out using Linomat $\mathrm{V}$ applicator with the help of nitrogen gas, the application was carried out under a constant flow rate to obtain a band length of $4 \mathrm{~mm}$ on the activated plate.

A twin trough chamber $(20 \times 10)$ was used for the development of the plates applied with samples and standards. Prior to development, the chamber was saturated with the mobile phase (ethyl acetate:methanol:toluene, 4:1:5) for $40 \mathrm{~min}$. The plates developed up to $80 \mathrm{~mm}$ in approximately $10 \mathrm{~min}$. The developed plates were then dried and scanned under $254 \mathrm{~nm}$ using TLC scanner IV. Evaluation of retention time of zonisamide and theophylline and their quantification was carried out using WinCats software.

Stock solutions of zonisamide and theophylline (internal standard) were prepared by dissolving $1 \mathrm{mg}$ of zonisamide and theophylline in $1 \mathrm{ml}$ of methanol individually. Calibration standards were prepared by diluting appropriate volume of zonisamide stock solution in drug-free human serum and further diluting it serially to obtain a calibration range of 5 to $80 \mu \mathrm{g} /$ band in human serum when applied on the plate. Three quality control samples of the concentrations 5, 20 and $40 \mu \mathrm{g} / \mathrm{band}$ were prepared separately using the stock solution and drug-free human serum. Stock solution of the internal standard was diluted to obtain working solution of $0.25 \mathrm{mg} / \mathrm{ml}$. About $10 \mu 1$ of working solution of internal standard was spiked in calibration standards and quality control samples, prior to extraction.

To $150 \mu 1$ of serum sample (calibration standards, quality control samples and patient samples), $10 \mu \mathrm{l}$ of working solution of internal standard was spiked and liquid-liquid extraction was carried out using $450 \mu \mathrm{l}$ of acetonitrile. The mixture was vortexed for $30 \mathrm{~s}$ and centrifuged at $21^{\circ}$ for $15 \mathrm{~min}$ at $6000 \mathrm{rpm}$. The supernatant was recovered and evaporated to dryness under stream of nitrogen using nitrogen gas evaporator. The residue was reconstituted using $60 \mu 1$ of methanol ${ }^{[11]}$.

The method was validated according to the International Conference on Harmonization guidelines using the parameters, specificity, linearity, accuracy and precision, limit of detection (LOD) and limit of quantification (LOQ), and robustness. Specificity of the method is determined to ensure that the developed method is able to quantitatively detect a specific analyte in presence of other components and related substances present in the sample matrix. This parameter confirms that there is no interference of the diluents, internal standards and other components at the retardation factor of zonisamide. Specificity was analysed by comparing chromatograms of blank serum samples, spiked serum samples, diluents used, internal standard and the reference standard. Further spectral analysis was performed to ensure the specificity of the spectra of reference standard and extracted zonisamide samples ${ }^{[11-15]}$. Also interference with other antiepileptic drugs such as phenytoin, phenobarbitone, oxcarbazepine, carbamazepine, sodium valproate, leviteracetam, lamotrigine and topiramate were checked with the $\mathrm{R}_{f}$ of zonisamide.

Linearity of the method is the ability of the method within a given range to obtain the test results i.e. the ratio of areas under curves of zonisamide and internal standard, which are directly proportional to the concentration in the analyte of the sample. A concentration range of 5 to $80 \mu \mathrm{g} / \mathrm{band}$ was used. The aliquots of each concentration were prepared, analysed in triplicate for 3 different days. The coefficient of correlation, standard deviation and regression of the line was analysed to assess linearity of calibration curve $\mathrm{e}^{[11-15]}$.

Precision is the indication of random error and is expressed in terms of relative standard deviation (RSD). Accuracy is closeness between the actual value and the analytical value obtained and is expressed as $\%$ recovery. Precision and accuracy of the method were assessed by performing replicate analyses of the 3 quality control samples $(5,40$ and $80 \mu \mathrm{g} /$ band, each concentration was prepared in three replicates, and each replicate was spotted thrice) against the calibration curve by carrying out the analytical run within the same day twice with $3 \mathrm{~h}$ interval (intra-day) and for $6 \mathrm{~d}$ (inter-day).

The LOD and LOQ were calculated with the help of a calibration curve of three samples having low concentrations of the drug. The LOD was calculated using the formula $3.3 \mathrm{\sigma} /$ slope of the calibration curve and LOQ was calculated using the formula $10 \mathrm{\sigma} / \mathrm{slope}$ of the calibration curve ${ }^{[11-15]}$.

Robustness is the capability of the method to remain unaffected by small changes in the method. Robustness 
was performed by changing the ratio of the mobile phase, altering the wavelength and altering the plate activation time. The altered mobile phase ratio used was ethyl acetate:methanol:toluene (3:2:5), ethyl acetate:methanol:toluene (5:1:4), the altered wavelength used was 250 and $260 \mathrm{~nm}$ and the plate were activated at $10^{\circ}$ and $120^{\circ}$. Robustness of the method was carried out in triplicates and its mean and $\%$ RSD were calculated ${ }^{[16,17]}$.

During the method development, mobile phase optimization was carried out by selecting proper solvents in different ratios and was finalized to be ethyl acetate:methanol:toluene (4:1:5) based on the resolution and stability with the saturation of $40 \mathrm{~min}$. Also the extraction method was developed by changing the optimized volume of acetonitrile used for extraction.

Zonisamide and the internal standard were well separated at retardation factor $\left(\mathrm{R}_{f}\right)$ of $0.61 \pm 0.2$ and $0.36 \pm 0.3$ (fig.1). The spectra of the serum sample spiked with zonisamide and internal standard and the spectra of their respective reference standards coincided indicating specificity of the method whereas blank plasma and diluents did not yield any peak at that $\mathrm{R}_{f}$ of both the analyte indicating no matrix interference (fig. 2). Additionally, other antiepileptic drugs did not show any interference at the $\mathrm{R}_{f}$ of zonisamide.

Precision and accuracy of the HPTLC method was determined by carrying out intra-day and inter-day analysis of the 3 quality control samples in replicate. The RSD for precision ranged from 2.83 to 3.27 and from 2.09 to 4.39 for intra and inter-day analyses, respectively (Table 1). Accuracy was calculated and was found between 97.41 to $111.13 \%$ and 97.52 to $115.23 \%$ for intra-day and inter-day analyses and is expressed as \% analyte recovery (Table 2 ).

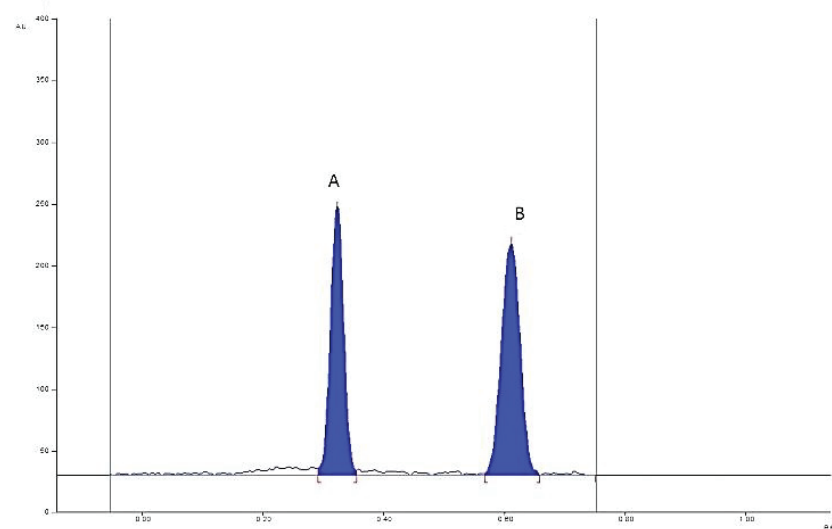

Fig. 1: HPTLC chromatogram of theophylline and zonisamide Separated peaks of $A$. theophylline at $\mathbf{R}_{f} \mathbf{0 . 3 6}$ and $B$. zonisamide at $\mathbf{R}_{f} \mathbf{0 . 6 1}$

March-April 2019

Indian Journal of Pharmaceutical Sciences

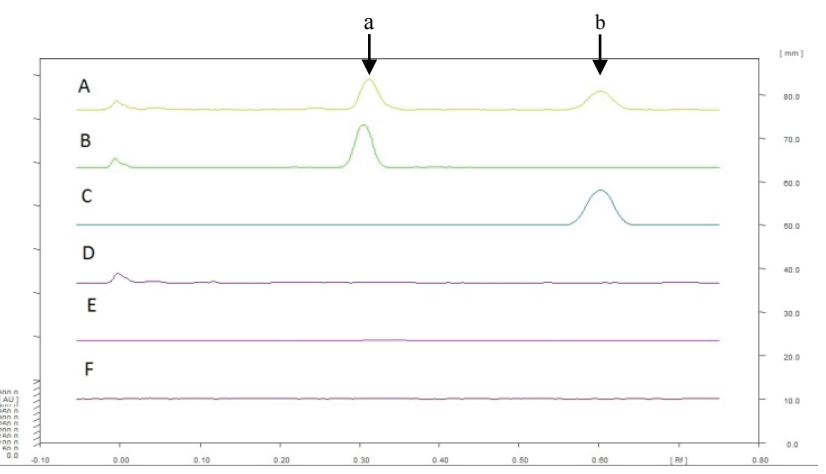

Fig. 2: Comparison of chromatograms

A. Human serum spiked with a. theophylline and b. zonisamide, B. a. theophylline, C. b. zonisamide, D. blank human serum, E. mobile phase and $\mathrm{F}$. diluent

TABLE 1: PRECISION OF ZONISAMIDE IN SERUM

\begin{tabular}{lcc}
\hline $\begin{array}{l}\text { Concentration } \\
(\mu \mathrm{g} / \mathrm{band})\end{array}$ & RSD intra-day & RSD inter-day \\
\hline 10 & 3.09 & 3.89 \\
40 & 3.27 & 4.39 \\
80 & 2.83 & 2.09 \\
\hline
\end{tabular}

TABLE 2: ACCURACY OF ZONISAMIDE IN SERUM

\begin{tabular}{lcc}
\hline $\begin{array}{l}\text { Concentration } \\
(\mu \mathrm{g} / \text { band) }\end{array}$ & $\begin{array}{c}\text { Calculated } \\
\text { concentration } \\
(\text { mean } \pm \text { SD) }\end{array}$ & Recovery \% \\
\hline Intra-day & & \\
\hline 10 & $11.10 \pm 0.61$ & 111.13 \\
40 & $42.14 \pm 0.69$ & 110.7 \\
80 & $88.83 \pm 1.08$ & 97.41 \\
Inter-day & & \\
\hline 10 & $11.18 \pm 1.03$ & 115.23 \\
40 & $41.59 \pm 0.72$ & 107.95 \\
80 & $89.01 \pm 1.89$ & 97.52 \\
\hline
\end{tabular}

The calibration curve was found to be linear with a regression Eqn. of $\mathrm{y}=52.5691( \pm 0.47)$ $X-181.5604( \pm 2.29)$, standard deviation of 3.09 and coefficient of correlation was found to be 0.991 . The lowest LOD and the lowest limit of quantification were found to be 1.07 and $3.15 \mu \mathrm{g} / \mathrm{band}$, respectively, which were well within in the therapeutic range. Robustness was carried out by altering one factor at a time while the other factors were kept constant. No large difference was observed in the peak area and the $\mathrm{R}_{f}$ of zonisamide. Table 3 indicates the robustness of the method.

The validated method was used for drug analysis on 30 epileptic patients, who were receiving oral zonisamide. Venous blood was collected aseptically and centrifuged at $1200 \mathrm{rpm}$ for $10 \mathrm{~min}$ to separate out the serum, which was then frozen at $-20^{\circ}$ till analysis. After carrying out the analysis of these 30 patients' samples, it was found out that the quantification values of 24 patient samples were well within the therapeutic range while 
TABLE 3: ROBUSTNESS

\begin{tabular}{lcc}
\hline Factors & Area & \% RSD \\
\hline $\begin{array}{l}\text { Change in mobile phase ratio } \\
\text { Ethyl acetate:methanol:toluene }\end{array}$ & 1246.70 & 2.34 \\
$\begin{array}{l}(3: 2: 5) \\
\text { Ethyl acetate:methanol:toluene }\end{array}$ & 1240.88 & 3.28 \\
$(5: 1: 4)$ & & \\
Change in wavelength & 1163.00 & 3.40 \\
$250 \mathrm{~nm}$ & 1211.25 & 2.69 \\
$260 \mathrm{~nm}$ & & \\
Change in plate activation & 1248.22 & 2.13 \\
temperature & 1259.11 & 2.95 \\
$100^{\circ}$ & & \\
$120^{\circ}$ & & \\
\hline
\end{tabular}

that of 2 patients were above the therapeutic range. The bands of the analyte were below the LOD in 4 patients. When the drug levels of these 4 patient samples were correlated with the medical history of the patients, it was found that one of the four patients, in whom the zonisamide level was below the detection limit, had stopped therapy $5 \mathrm{~d}$ previously while 3 patients had history of poor compliance.

Maintaining effective concentrations of antiepileptic drugs in blood is very important to attain proper seizure control in epileptic patients. Changes in the blood concentration of an antiepileptic drug can lead to harmful side effects. Therapeutic drug monitoring aids in monitoring the blood concentration of the antiepileptic drug, which is clinically useful to assess patient adherence and to adjust dosing when required, optimizing seizure control and minimizing toxic effects. Zonisamide shows pharmacokinetic variability when administered concomitantly with other antiepileptic drugs, especially those with enzyme inducing properties and also in case of renal impairment and thus it becomes important to perform therapeutic drug monitoring to maintain drug levels.

A wide range of approaches to assess blood concentration of zonisamide have been proposed, however these techniques are very expensive and are not accessible to every patient. Considering the effectiveness of this new antiepileptic drug, it is important that therapeutic drug monitoring of zonisamide be carried out cost effectively so that all patients can easily manage regular monitoring and dose adjustment. Curiously enough, there are very few reports on therapeutic drug monitoring using HPTLC, which is a comparatively less expensive technique. Hence, a method for the determination of serum zonisamide levels using HPTLC was developed, which was found to be simple, reliable and cheap. Simple, as it involved simple liquid-liquid extraction using acetonitrile. The single step extraction did not jeopardize the accuracy and specificity. In fact, this method gave better resolution as there was no matrix interference. Using theophylline as an internal standard for the determination of zonisamide concentration made the method more reliable as it gave a well separated peak without any matrix interference. Robustness indicated that the method is stable over small changes. The mobile phase used gave proper resolution of the analyte and the internal standard and was also used in small volumes compared to the HPLC technique, which further reduced the cost.

When the results of the developed and validated method were compared to those obtained using high performance liquid chromatography (HPLC), HPTLC and gas chromatography, there were no significant difference in the accuracy and precision, the only limitation was that the LOQ and LOD were higher in the present study as compared to those obtained using the HPLC method. The lowest LOQ in HPLC documented in literature was approximately $0.1 \mu \mathrm{g} / \mathrm{ml}^{[18,19]}$ while in the present study, it was $3.15 \mu \mathrm{g} / \mathrm{ml}$. However, the LOQ of this HPTLC method is well below the therapeutic range, which is 10 to $40 \mu \mathrm{g} / \mathrm{ml}$ and therefore, could be used reliably to carry out therapeutic drug monitoring. The HPTLC method allows analysis of 10 samples at the same time, thus reducing the turnaround time and earlier reporting of patient samples. Thus the developed and validated HPTLC method is simple and reliable to perform therapeutic drug monitoring of zonisamide.

\section{Acknowledgements:}

We would like to thank Sun Pharmaceuticals Industries Limited, Mumbai India for their kind donation of zonisamide sample.

\section{REFERENCES}

1. Dash A, Ravat S, Srinivasan AV, Shetty A, Kumar V, Achtani $\mathrm{R}$, et al. Evaluation of safety and efficacy of Zonisamide in adult patients with partial, generalized, and combined seizures: an open labeled, non-comparative, observational Indian study. Ther Clin Risk Manag 2016;12:327.

2. $\mathrm{Ma}^{3} \mathrm{ek}$ R, Piskorska B, Czuczwar SJ. Zonisamide: a new antiepileptic drug. Pol J Pharmacol 2003;55:683-9.

3. Nair AB, Jacob S. An updated overview of therapeutic drug monitoring of recent antiepileptic drugs. Drugs R D 2016;16:303-16.

4. Leppik IE. Zonisamide: chemistry, mechanism of action, and pharmacokinetics. Seizure 2004;13:S5-9.

5. Schmidt D, Jacob R, Loiseau P, Deisenhammer E, Klinger $\mathrm{D}$, Despland A, et al. Zonisamide for add-on treatment of refractory partial epilepsy: a European double-blind trial. Epilepsy Res 1993;15(1):67-73. 
6. Yanai S, Hanai T, Narazaki O. Treatment of infantile spasms with zonisamide. Brain Dev 1999;21(3):157-61.

7. Makino K, Goto Y, Sueyasu M, Futagami K, Kataoka Y, Oishi R. Micellarelectrokinetic capillary chromatography for therapeutic drug monitoring of zonisamide. J Chromatogr B Biomed Sci Appl 1997;695(2):417-25.

8. Greiner-Sosanko E, Giannoutsos S, Lower DR, Virji MA, Krasowski MD. Drug monitoring: simultaneous analysis of lamotrigine, oxcarbazepine, 10-hydroxycarbazepine, and zonisamide by HPLC-UV and a rapid GC method using a nitrogen-phosphorus detector for levetiracetam. J Chromatogr Sci 2007;45(9):616-22.

9. Yoshida T, Imai K, Motohashi S, Hamano SI, Sato M. Simultaneous determination of zonisamide, carbamazepine and carbamazepine-10, 11-epoxide in infant serum by highperformance liquid chromatography. J Pharm Biomed Anal 2006;41(4):1386-90.

10. Greiner-Sosanko E, Lower DR, Virji MA, Krasowski MD. Simultaneous determination of lamotrigine, zonisamide, and carbamazepine in human plasma by high-performance liquid chromatography. Biomed Chromatogr 2007;21(3):225-8.

11. Antonilli L, Brusadin V, Filipponi F, Guglielmi R, Nencini P. Development and validation of an analytical method based on high performance thin layer chromatography for the simultaneous determination of lamotrigine, zonisamide and levetiracetam in human plasma. J Pharm Biomed Anal 2011;56(4):763-70.

12. Rashmin P, Mrunali P, Nitin D, Nidhi D, Bharat P. HPTLC method development and validation: strategy to minimize methodological failures. J Food Drug Anal 2012;20(4):794804.
13. Shabir GA, John Lough W, Arain SA, Bradshaw TK. Evaluation and application of best practice in analytical method validation. J Liquid Chromatogr Relat Technol 2007;30(3):311-33.

14. Ferenczi-Fodor K, Végh Z, Nagy-Turak A, Renger B, Zeller $M$. Validation and quality assurance of planar chromatographic procedures in pharmaceutical analysis. J AOAC Int 2001;84(4):1265-76

15. Ferenczi-Fodor K, Renger B, Végh Z. The frustrated reviewer-recurrent failures in manuscripts describing validation of quantitative TLC/HPTLC procedures for analysis of pharmaceuticals. J Planar Chromatogr 2010;23(3):173-9.

16. Kaur I, Wakode S, Singh HP. Development and validation of a stability-indicating high performance thin layer chromatography (HPTLC) method for estimation of canagliflozinin bulk and pharmaceutical dosage form. J Appl Pharm Sci 2016;6(5):051-7.

17. Jain A, Parashar AK, Nema RK, Narsinghani T. High Performance Thin Layer Chromatography (HPTLC): A Modern Analytical Tool for Chemical Analysis. Curr Res Pharm Sci 2014;4(01):08-14.

18. Yeniceli D. Development and validation of a simple and efficient HPLC method for the determination of zonisamide in pharmaceuticals and human plasma. J Anal Chem 2013;68(5):436-43.

19. Dupouey J, Doudka N, Belo S, Blin O, Guilhaumou R. Simultaneous determination of four antiepileptic drugs in human plasma samples using an ultra-high-performance liquid chromatography tandem mass spectrometry method and its application in therapeutic drug monitoring. Biomed Chromatogr 2016;30(12):2053-60. 